\title{
Children and Youth in Low- and Middle-Income Countries: Toward An Integrated Developmental and Intervention Science
}

\author{
Alice J. Wuermli, ${ }^{1}$ Carly C. Tubbs, ${ }^{2}$ Anne C. Petersen, ${ }^{3}$ and J. Lawrence Aber ${ }^{2}$ \\ ${ }^{1}$ University of California, Davis, ${ }^{2}$ New York University, and ${ }^{3}$ University of Michigan
}

ABSTRACT_In this article, we discuss the need for and value of an integrated developmental intervention science in low- and middle-income countries (LAMICs), and the extent to which this integration of basic developmental science has influenced research on child and youth development interventions in these countries. We also consider how a global developmental science can be advanced by capitalizing on the opportunities provided by cross-cultural, interdisciplinary, experimental, and mixed methods approaches. We argue that designing and evaluating human developmental interventions in LAMICs present an opportunity for both research and policy. Testing contextualized developmental approaches through rigorously evaluated interventions yields the promise of finding more

Alice J. Wuermli, University of California, Davis; Carly C. Tubbs, New York University; Anne C. Petersen, University of Michigan; J. L. Aber, New York University.

The authors were invited by the Society for Research on Child Development (SRCD) to design and host a preconference on Interventions for Children and Youth in Low- and Middle-Income Countries: An Opportunity for Developmental Science, on April 17, 2013, in Seattle, WA. This article draws together lessons learned from the preconference, as well as our work over the past year developing an international research center at New York University (Global TIES for Children) and a global network (the Human Development Intervention Network) devoted to this topic. We thank SRCD and the Jacobs Foundation for cosponsoring the preconference; the preconference presenters, case study leaders, and participants for their invaluable contributions; all of our colleagues, whose work continues to inspire us; and Hirokazu Yoshikawa (New York University) and Jennifer Lansford (Duke University), who provided invaluable comments on drafts of this article.

Correspondence concerning this article should be addressed to Alice J. Wuermli, Department of Human Ecology, University of California, Davis, Davis, CA 95616; e-mail: awuermli@ucdavis.edu.

(C) 2015 The Authors

Child Development Perspectives @ 2015 The Society for Research in Child Development DOI: $10.1111 /$ cdep. 12108 poverful and scalable solutions that can reach more children and youth worldwide. Moreover, a theoretically rich and methodologically rigorous understanding of how interventions effect change in diverse countries and contexts can contribute to the development of a truly global developmental science.

KEYWORDS-low- and middle-income countries; intervention evaluation; policy; early childhood development; child development; youth development; adolescent development; culture; global

\footnotetext{
"If you want truly to understand something, try to change it." Attributed to Kurt Lewin (1, p. 31)
}

All too often, adversities such as poverty and economic shocks, violence and war, and poor health and nutrition systems systematically undermine children's - and consequently, their nations - capacity to thrive. In particular, in low- and middleincome countries ${ }^{1}$ (LAMICs), such adversities can overwhelm people and institutions already struggling with limited resources. To address these adversities, organizations in both national and international arenas are designing, implementing, and rigorously evaluating policies and programs to combat family and community poverty, buffer children and families from the effects of violence and war, promote access to education, and improve children's health and development. However, these programswhich have cost billions of dollars - have yet to reach optimal effectiveness for millions of children worldwide.

Along with a growing group of researchers, practitioners, and policymakers, we propose that incorporating principles from human developmental science into the design, implementation,

${ }^{1}$ Low-income countries are defined as having a Gross National Income per capita (GNI/cap) of $\$ 1,045$ or lower; middle-income countries are those with GNI/ cap above $\$ 1,045$ and below $\$ 12,746$ in 2013 . For details, see http://data.worldbank.org/about/country-and-lending-groups. 
and evaluation of programs and policies in LAMICs can improve their impact, cost-effectiveness, sustainability, and scalability (2). In this article, we discuss how integrating intervention research and basic developmental science more effectively can improve research, programs, and policies. We also examine to what extent basic developmental science, especially such science carried out by local researchers, has influenced intervention and evaluation designs in LAMICs. And we consider how capitalizing on the opportunities provided by cross-cultural, interdisciplinary, experimental, and mixed methods approaches can advance a global developmental science.

It is difficult in an article this short to adequately cover the fields of developmental science and developmental intervention research from LAMICs. For developmental interventions in LAMICs, we focused on examples with available experimental and quasi-experimental evidence. We also under-referenced the cross-cultural, contextualized developmental literature from LAMIC. In cases in which several sources existed, we included only one that exemplified the others.

\section{CREATING THE "PERFECT BLEND": OPTIMIZING DEVELOPMENTAL AND INTERVENTION SCIENCE THROUGH INTEGRATION}

Developmental science and intervention science reinforce one another. Our ability to understand children across time and contexts (3), and to apply that knowledge to programs and policies to promote children's development, has yielded more effective interventions that provide evidence of contextually embedded developmental processes. Developmental science helps identify and measure environmental and developmental mechanisms that are promising targets for interventions. It also provides information on how programs operate differently for different people in different contexts. Such variation can stem from interindividual differences in intraindividual change and insensitivity to environmental risk and protective factors, and the nested nature of human development.

Progressively, intervention research is designed to investigate causal mechanisms that explain the impacts of interventions and sources of heterogeneity in those impacts. Designed properly, intervention evaluations in which participants are assigned randomly to either the treatment or control condition enable developmentalists to test hypotheses about causal mediation (4). And conducting this research across cultures allows us to test the applicability and meaningfulness of developmental theories, concepts, and mechanisms for different populations.

\section{WHERE HAS DEVELOPMENTAL THINKING PENETRATED INTERVENTION SCIENCE IN LAMICS AND WHERE ARE WE LAGGING?}

Recognizing such arguments, evaluations of interventions in the United States and other member states of the Organization for
Economic Cooperation and Development (OECD) increasingly incorporate theory, measures, and methods from developmental science, shedding light on the underlying mechanisms and processes through which interventions effect change. However, intervention science in LAMICs has not yet sufficiently integrated such approaches. Common measures used (e.g., anthropometrics, school attendance), while technically accurate and easily observable, leave much to be desired in terms of their effects on the full range of children's development across domains. Recently, governments and international development organizations have been interested in moving a global human development intervention science toward stronger developmentally infused theories of change that can capture these complex processes and mechanisms. For example, global early childhood development integrates developmental theories, including concepts of risk and protection, cross-domain influences, and developmental specificity of targets, from the prenatal period to age 8 (5). While nutrition is a common focus, evaluated programs now often take a more comprehensive approach, combining emphases on children's survival (e.g., health care, immunizations, nutrition) with early education, psychosocial and cognitive stimulation, and parenting (6). Outcomes have expanded beyond language and numeracy (e.g., executive functions), and studies now compare the effects of different approaches (nutrition vs. psychosocial stimulation) and combinations of these approaches (7). Yet, scientists and policymakers need to understand more completely the comparative cost-effectiveness, depending on when and where, under what circumstances, and how such services are delivered (8).

Recently, researchers have promoted more developmentally appropriate intervention and research designs, but the available evidence for certain contexts, populations, and outcomes varies (9). Particularly striking is the dearth of evidence on developmental interventions in places and among populations affected by conflict, including refugee camps (10).

Most interventions targeting middle childhood are focused on education. Researchers have progressed in the last two decades toward reaching the United Nations Millennium Development Goal of universal enrollment in primary school, which has resulted in many intervention studies measuring attendance or enrollment rates. However, researchers, practitioners, and governments are concerned about actual learning outcomes, which are reflected in the ongoing negotiations about the United Nations' post-2015 Sustainable Development Goals (11). These include not only literacy and numeracy but also other dimensions of learning key to success, such as socioemotional learning. ${ }^{2}$ While OECD countries have shifted toward improving learning through interventions in learning environments (12), these are infrequent in LAMICs (13).

\footnotetext{
${ }^{2}$ For more information, see the report of the Learning Metrics Task Force at http://www.brookings.edu/research/reports/2013/09/learning-metrics-task-forceuniversal-learning.
} 
While Heckman and colleagues have influenced a shift in thinking about the skills needed to find and hold gainful employment, policy recommendations have focused on early investments (14). Concurrently, though, we should be thinking about adolescents' developmental needs and capacity for change. Thus, as Verma (15) points out, we need to understand more fully how to foster life skills in adolescents and young adults (such skills are known in developmental circles as socioemotional skills or self-regulation, and in economics and business as soft or noncognitive skills). Most programs for disadvantaged and unemployed youth target only employment, and typically resemble public works programs or vocational training rather than enhancing development more broadly (16). Adolescent reproductive health and risky behaviors are other areas that have been studied (17).

\section{LOOKING AHEAD AT DEVELOPMENTAL INTERVENTION SCIENCE IN LAMICS}

We now highlight some of the most advanced human development interventions, evaluation designs, methods, and measurement approaches implemented in LAMICs.

\section{What Are the Features of Developmentally and Culturally} Appropriate Interventions and Evaluations?

Rao and Woolcock's work on how history and culture affect politics, institution building, and policymaking has begun to penetrate the way international development agencies involved in policymaking think and practice (18), as well as the crosscultural study of child development (19). Local governments, nongovernmental organizations (NGOs), and researchers who have spent much time in the countries in which they are working have developed culturally and developmentally informed interventions. However, many of these initiatives lack rigorous evidence on cost-effectiveness, or have not been implemented at scale, two practices recommended and sometimes required by lending institutions and in the national policymaking process.

Being culturally and contextually appropriate requires attention to local norms and perceptions about children's development, including perceptions and practices related to parenting and family structure (20). Developmental appropriateness refers to a child's capacity at a given stage of development, which may vary depending on local beliefs and practices (21). Thus, researchers should seek to develop interventions that are age appropriate and culturally sensitive: Interventions and their evaluations should be informed by and inform local developmental science by local scholars and practitioners. The Pakistani version of the Care for Child Development module represents a rigorously evaluated successful adaptation (e.g., it uses an existing health service system of paraprofessionals where skilled nurses are scarce, delivered through home visits exclusively by female workers in a context in which women's interactions with men outside their families are restricted; 6).
One way to encourage cultural and contextual appropriateness is to use a community-based approach to designing and implementing programs; community-based strategies also yield promise in terms of sustainability in settings where resources are limited. For example, in a community-based preschool program in Mozambique, communities managed and supervised the preschool centers. Groups were eligible to receive support from the NGO implementing the program if they could provide the space, use local construction materials, do all the construction labor, and establish a committee to engage with parents and facilitate construction and maintenance (22). (Another interesting example comes from Cambodia; 23.)

\section{How Do We Ensure That Intervention Research is Developmentally Appropriate and Culturally Sensitive and Relevant?}

Most research in human development has been conducted in OECD countries and, consequently and disproportionately, on White, middle-class groups (24). Our tested theories, concepts, and measures reflect this narrow focus and are in many cases inadequate for capturing the realities of most of the world's children. This limits the claims we can make about the universality of many developmental theories and concepts, and constrains the ability of developmental science to inform policy in LAMICs.

At the same time, we have progressed toward hypothesizing the underlying mechanisms and processes of development that may apply more broadly $(25,26)$. Most contemporary theories of human development are rooted in bioecological frameworks that indicate a confluence of dynamic processes between the developing individual and the many levels of human ecology (3). Basic developmental research often lacks the power to make strong causal statements, but can identify the potential mechanisms worth exploring. For instance, in an evaluation of a school-based intervention, changes in effective teaching causally mediated improvements in children's learning, a link previously identified in correlational studies (4).

In LAMICs, economists have been at the forefront of researching poverty and evaluating programs using rigorous experimental and quasi-experimental designs. The disciplinary emphasis on causal inference has supported the development and adaptation of experimental and quasi-experimental methods. Randomized controlled trials allow us to approximate a situation of an exogenous variable through random assignment to treatment. Yet the endogeneity of behavioral choices poses difficulties for causal inference (27). Fixed effects at the individual, family, school, or community level are commonly used to account for the variance that is caused by unobserved factors at these levels. However, this is akin to a black-box approach in which we gain no insights into the mechanisms driving the results (27).

In addition, while randomized evaluation designs offer the highest standard of evidence for the causal impact of an intervention, the specifics of a particular context raise questions 
about the external validity of the findings (28). Thus, researchers must account for contextual influences and variation. Insights from developmental science and other disciplines demonstrate the usefulness of hierarchical linear as well as structural models in which such relationships are tested explicitly. Yet the best models are of little use without data to test them. Local developmental researchers need to inform decisions in intervention studies in LAMICs about what to measure, when, where, and how.

Measures need to be adapted to and validated in a particular cultural context. For instance, several African languages distinguish between cognitive speed and social responsibility, a combination of the two being socially desirable (29). The work suggests the importance of including social responsibility as a dimension of intelligence if research is to resonate with local understandings of child development, and "achieve recognition as a source of guidance for African families, service practitioners, and policymakers" (29, p. 126). Local researchers can "more sensitively draw on ethnotheories and life-journeys from diverse cultures" to describe variations in cultural supports, constraints, and curricula to human development (30, p. 2). Where local capacity is lacking, we need to invest in building it. This capacity will be crucial when balancing the demands of research and the need for interventions (31). In addition, we need to find ways to share and validate research findings with the local communities that are to benefit from the policy implications drawn from the work (29).

In some cases, intensive developmental and epidemiological studies have laid the groundwork to inform the design and evaluation of interventions. Yet we also need systematic approaches that develop, adapt, and validate measures and concepts that allow for cross-cultural comparisons and conclusions. Researchers have developed and validated culturally grounded measures and assessments of children's (32) and youth's (33) outcomes. Such tools need to be open source to encourage widespread use and allow for cross-cultural comparisons. However, the crosscultural comparability of measures and methods to support this may conflict with validity and within-cultural meaningfulness (9).

Research in LAMICs needs to expand to include more recent advances in developmental science (34). Researchers in developmental neuroscience, behavioral endocrinology, and epigenetics have begun to illuminate the interplay between context and an individual's biology, as well as how this affects behavior and well being. Intervention studies in OECD countries have started to account for biological processes, finding that they explain some of the heterogeneity in program impacts (35). Yet intervention studies in LAMICs have yet to incorporate these methods and measures. Biology often provides more information about processes under the skin, illuminating, for example, resilience and how it can be created or changed. We need to understand more fully how the effectiveness of interventions is mediated or moderated by individual physiology, how interventions alter physiological processes and behavior, and how physiology can improve our understanding of the underlying processes affecting both health and behavior.

Several researchers have argued for rigorous mixed methods approaches $(36,37)$. Mixed methods allow us to assess quantitative aspects of program effectiveness and inferences of causality, as well as qualitative aspects such as cultural appropriateness, complicated impact pathways, unanticipated consequences, and reasons for a program's effectiveness. In other words, mixed methods can help us understand why interventions work the way they do (38). Mixed methods can inform our hypotheses and intervention designs, and can help us interpret the quantitative data, as well as provide information about social and institutional change. Thus, combining the most powerful quantitative and qualitative tools will support the development and validation of measures, theories, and intervention and research designs, and can enhance our understanding of the contextual variables that contribute to the successful scaling of programs.

\section{What Are the Challenges of Scaling Evidence-Based Interventions?}

Ultimately, improvements at the country, regional, and global level will be achieved only by scaling programs successfully. Yet, as Friedman (28) highlighted, lack of external validity may cause interventions to fail to show impacts when implemented in different contexts or at greater scale (39). We argue that contextualized research will allow us to take evidence-based interventions to scale through a deeper understanding of the conditions required for a program to be effective, and that scaling in and of itself changes the context, which in turn determines the program's success or failure (40). This requires careful implementation science and continuous quality improvement models (41). The process of scaling and expanding services invariably changes contextual parameters at the sociocultural and systems levels relevant to a program's effectiveness; services and service-delivery systems need to be adapted continuously to account for these contextual changes.

\section{DEVELOPING A TRULY GLOBAL DEVELOPMENTAL SCIENCE}

We want to harness the best of developmental science to advance intervention science, and harness the best of intervention science to advance developmental science. Designing and evaluating human development interventions in LAMICs is and should be seen as an opportunity. Testing contextualized developmental approaches through interventions will yield more powerful and scalable solutions that can reach more children and youth.

Developing local capacity for research as well as intervention design and implementation may take time. In the meantime, the human development community needs to pool resources available to advance the field by investing in careful reviews of the 
evidence, writing detailed accounts of the implementation process and monitoring information, communicating openly and sharing good and bad experiences among stakeholders, and engaging with policymakers and representatives of civil society. Funders should insist on rigorous evaluations, and that researchers use culturally appropriate and developmentally valid measures and assessments; NGOs should be open to independent evaluation; and researchers should collaborate across disciplines to achieve the highest standard of evaluation and basic developmental science. Finally, researchers need to communicate findings in ways that ensure widespread dissemination (e.g., through blogs ${ }^{3}$ and working papers, ${ }^{4}$ approaches that are well established at institutions such as the World Bank).

In summary, we need a large, intensive effort to achieve the goals we have outlined in this article. Despite the size of the task, the goals are worth the investment. A theoretically rich and methodologically rigorous understanding of the impact of interventions on children and youth in low- and middle-income countries will contribute to the development of a developmental science that is truly global.

\section{REFERENCES}

1. Tolman, C. W. (1996). Problems of theoretical psychology. North York, ON: Captus Press.

2. Wuermli, A. J., Hempel, K., Aber, J. L., \& Lundberg, M. (2012). Policies to protect and promote young people's development during crisis. In M. Lundberg \& A. J. Wuermli (Eds.), Children and youth in crisis: Protecting and promoting human development in times of economic shocks (pp. 229-277). Washington, DC: World Bank.

3. Bronfenbrenner, U., \& Morris, P. A. (2006). The bioecological model of human development. In W. Damon \& R. M. Lerner (Eds.), Handbook of child psychology: Theoretical models of human development (Vol. 1, 6th ed., pp. 793-828). Hoboken, NJ: Wiley.

4. Jones, S. M., Brown, J., \& Aber, J. L. (2014). Testing multi-level causal processes in complex settings-level interventions: The mediating role of classroom quality in a socio-emotional learning program. Unpublished manuscript.

5. Britto, P. R., Engle, P. L., \& Super, C. M. (2013). Handbook of early childhood development research and its impact on global policy. New York, NY: Oxford University Press.

6. Yousafzai, A. K., Rasheed, M. A., Rizvi, A., Armstrong, R., \& Bhutta, Z. A. (2014). Effect of integrated responsive stimulation and nutrition interventions in the Lady Health Worker programme in Pakistan on child development, growth, and health outcomes: A cluster-randomised factorial effectiveness trial. Lancet, 384, 12821293. doi:10.1016/s0140-6736(14)60455-4

7. Grantham-McGregor, S. M., Fernald, L. C. H., Kagawa, R. M. C., \& Walker, S. (2014). Effects of integrated child development and nutrition interventions on child development and nutritional status. Annals of the New York Academy of Sciences, 1308, 11-32. doi: 10.1111/nyas.12284

8. Ruel, M. T., \& Alderman, H. (2013). Nutrition-sensitive interventions and programmes: How can they help to accelerate progress in

${ }^{3}$ http://blogs.worldbank.org/impactevaluations/.

${ }^{4}$ http://elibrary.worldbank.org/page/wb-working-papers. improving maternal and child nutrition? Lancet, 382, 536-551. doi:10.1016/S0140-6736(13)60843-0

9. Marfo, K. (2013, April). Education interventions and cognitive development in early to middle childhood. Preconference on Interventions for Children and Youth in Low- and Middle-Income Countries: New Opportunities and Challenges for Developmental Science, Seattle, WA.

10. Burde, D., Kapit, A., Wahl, R., Guven, O., \& Skarpeteig, M. (2014). Education in emergencies: A review of theoretical and empirical research. Unpublished manuscript.

11. Burnett, N., \& Felsman, C. (2012). Post-2015 education MDGs. London, UK: Results for Development Institute \& Overseas Development Institute.

12. Cappella, E., Hamre, B. K., Kim, H. Y., Henry, D. B., Frazier, S. L., Atkins, M. S., \& Schoenwald, S. K. (2012). Teacher consultation and coaching within mental health practice: Classroom and child effects in urban elementary schools. Journal of Consulting and Clinical Psychology, 80, 597-610. doi:10.1037/a0027725

13. Torrente, C., Johnson, B., Starkey, L., Seidman, E., Shivshanker, A., Annan, J., \& Aber, J. L. (2014). Improving school environments and student well-being: Impacts after one year of a school-based intervention in the Democratic Republic of the Congo. Unpublished manuscript.

14. Heckman, J. J., \& Masterov, D. V. (2007). The productivity argument for investing in young children. Applied Economic Perspectives and Policy, 29, 446-493. doi:10.1111/j.1467-9353.2007.00359.x

15. Verma, S. (2013, April). Life skills interventions for psychosocial development in adolescents. Preconference on Interventions for Children and Youth in Low- and Middle-Income Countries: New Opportunities and Challenges for Developmental Science, Seattle, WA.

16. Betancourt, T. S., Newnham, E. A., Hann, K., McBain, R., Akinsulure-Smith, A., Weisz, J., ... Hansen, N. (2014). Addressing the consequences of violence and adversity: The development of a group mental health intervention for war-affected youth in Sierra Leone. In J.-P. Raynaud, S. Shur-Fen Gau, \& M. Hodes (Eds.), From research to practice in child and adolescent mental health (pp. 157-177). New York, NY: Rowman \& Littlefield.

17. Jewkes, R., Nduna, M., Levin, J., Jama, N., Dunkle, K., Puren, A., \& Duvvury, N. (2008). Impact of stepping stones on incidence of HIV and HSV-2 and sexual behaviour in rural South Africa: Cluster randomised controlled trial. British Medical Journal, 337, a506. doi:10.1136/bmj.a506

18. Woolcock, M., Szreter, S., \& Rao, V. (2011). How and why does history matter for development policy? The Journal of Development Studies, 47, 70-96. doi:10.1080/00220388.2010.506913

19. LeVine, R. A. (2007). Ethnographic studies of childhood: A historical overview. American Anthropologist, 109, 247-260. doi:10.1525/ AA.2007.109.2.247.

20. Nsamenang, A. B., \& Lamb, M. E. (1995). The force of beliefs: How the parental values of the Nso of Northwest Cameroon shape children's progress toward adult models. Journal of Applied Developmental Psychology, 16, 613-627.

21. Super, C. M., Harkness, S., Barry, O., \& Zeitlin, M. (2011). Think locally, act globally: Contributions of African research to child development. Child Development Perspectives, 5, 119-125. doi:10.1111/j.1750-8606.2011.00166.x

22. Martinez, S., Naudeau, S., \& Pereira, V. (2012). The promise of preschool in Africa: A randomized impact evaluation of early childhood development in rural Mozambique. Washington, DC: World Bank Group \& Save the Children. 
23. Bouguen, A., Filmer, D., Macours, K., \& Naudeau, S. (2013). Impact evaluation of three types of early childhood development interventions in Cambodia. Policy Research Working Paper 6540. Washington, DC: World Bank.

24. Henrich, J., Heine, S. J., \& Norenzayan, A. (2010). Most people are not WEIRD. Nature, 466, 29.

25. Sameroff, A. J., \& MacKenzie, M. J. (2003). Research strategies for capturing transactional models of development: The limits of the possible. Development and Psychopathology, 15, 613-640. doi:10.1017/S0954579403000312

26. Tseng, V., \& Seidman, E. (2007). A systems framework for understanding social settings. American Journal of Community Psychology, 39, 217-228. doi:10.1007/s10464-007-9101-8

27. Behrman, J. (2013, April). One economist's perspective on some important estimation issues. Preconference on Interventions for Children and Youth in Low- and Middle-Income Countries: New Opportunities and Challenges for Developmental Science, Seattle, WA.

28. Friedman, J. (2013, April). Implementing and evaluating interventions in low- and middle-income countries: A few guiding principles. Preconference on Interventions for Children and Youth in Low- and Middle-Income Countries: New Opportunities and Challenges for Developmental Science, Seattle, WA.

29. Serpell, R. (2011). Social responsibility as a dimension of intelligence, and as an educational goal: Insights from programmatic research in an African society. Child Development Perspectives, 5, 126-133. doi:10.1111/j.1750-8606.2011.00167.x

30. Nsamenang, B. (2000). Issues in indigenous approaches to developmental research in Sub-Saharan Africa. International Society for the Study of Behavioural Development Newsletter, 37, 1-3.

31. Smith, S. K. (2013, April). Designing, implementing, and evaluating interventions in low- and middle-income countries. Preconference on Interventions for Children and Youth in Low- and Middle-Income Countries: New Opportunities and Challenges for Developmental Science, Seattle, WA.

32. Gladstone, M., Lancaster, G. A., Umar, E., Nyirenda, M., Kayira, E., van den Broek, N. R., \& Smyth, R. L. (2010). The Malawi Developmental Assessment Tool (MDAT): The creation, validation, and reliability of a tool to assess child development in rural African settings. PLoS Medicine, 7, el000273. doi:10.1371/journal.pmed. 1000273
33. Ng, L. C., Kanyanganzi, F., Munyanah, M., Mushashi, C., \& Betancourt, T. S. (2014). Developing and validating the youth conduct problems scale-Rwanda: A mixed methods approach. PLoS ONE, 9, e100549. doi:10.1371/journal.pone.0100549

34. Sommer, S. (2013). Commentary: Leapfrogging as a principle for research on children and youth in majority world settings. Journal of Research on Adolescence, 23, 187-188. doi:10.1111/j.15327795.2012.00835.x

35. Bakermans-Kranenburg, M. J., Van Ijzendoorn, M. H., Mesman, J., Alink, L. R., \& Juffer, F. (2008). Effects of an attachment-based intervention on daily cortisol moderated by dopamine receptor D4: A randomized control trial on 1- to 3-year-olds screened for externalizing behavior. Developmental Psychopathology, 20, 805-820. doi:10.1017/s0954579408000382

36. Adato, M. (2011). Combining quantitative and qualitative methods for program monitoring and evaluation: Why are mixed-method designs best? Special Series on the Nuts \& Bolts of M\&E Systems. PREMnotes, 9. Washington, DC: The World Bank.

37. Huston, A. C., Duncan, G. J., \& Yoshikawa, H. (2015). Mixed methods in the science of understanding anti-poverty policies for families with children: Four case studies. In C. M. Hay (Ed.), Methods that matter. unpublished manuscript.

38. Adato, M. (2013, April). Qualitative and mixed methods in interdisciplinary Program evaluation. Preconference on Interventions for Children and Youth in Low- and Middle-Income Countries: New Opportunities and Challenges for Developmental Science, Seattle, WA.

39. Morduch, J., Ravi, S., \& Bauchet, J. (2013). Substitution bias and external validity: Why an innovative anti-poverty program showed no net impact. PRIMCED Discussion Paper Series, No. 44. Tokyo, Japan: Hitotsubashi University.

40. Dodge, K. A. (2011). Context matters in child and family policy. Child Development, 82, 433-442. doi:10.1111/j.1467-8624.2010. 01565.x

41. Yoshikawa, H. (2013, April). Five kinds of research to improve the quality of developmental contexts for children in low- and middle-income countries. Preconference on Interventions for Children and Youth in Low- and Middle-Income Countries: New Opportunities and Challenges for Developmental Science, Seattle, WA. 